\title{
A pilot survey of stellar tidal streams in nearby spiral galaxies
}

\author{
David Martínez-Delgado ${ }^{1,2}$, R. Jay Gabany ${ }^{3}$, Jorge Peñarrubia ${ }^{4}$, Hans-Walter Rix ${ }^{2}$, \\ Steven R. Majewski ${ }^{5}$, Ignacio Trujillo ${ }^{1}$ and Michael Pohlen ${ }^{6}$ \\ ${ }^{1}$ Instituto de Astrofísica de Canarias, La Laguna, Spain \\ ${ }^{2}$ Max-Planck-Institute fur Astronomie, Heidelberg, Germany \\ ${ }^{3}$ Black Bird Observatory, New Mexico, USA \\ ${ }^{4}$ Institute of Astronomy, Cambridge, UK \\ ${ }^{5}$ Department of Astronomy, University of Virginia, USA \\ ${ }^{6}$ Cardiff University, UK
}

\begin{abstract}
Within the hierarchical framework for galaxy formation, merging and tidal interactions are expected to shape large galaxies to this day. While major mergers are quite rare at present, minor mergers and satellite disruptions - which result in stellar streams - should be common, and are indeed seen in both the Milky Way and the Andromeda Galaxy. As a pilot study, we have carried out ultra-deep, wide-field imaging of some spiral galaxies in the Local Volume, which has revealed external views of such stellar tidal streams at unprecedented detail, with data taken at small robotic telescopes (0.1-0.5-meter) that provide exquisite surface brightness sensitivity. The goal of this project is to undertake the first systematic and comprehensive imaging survey of stellar tidal streams, from a sample of $\sim 50$ nearby Milky-Waylike spiral galaxies within $15 \mathrm{Mpc}$, that features a surface brightness sensitivity of $\sim$ $30 \mathrm{mag} / \mathrm{arcsec}^{2}$. The survey will result in estimates of the incidence, size/geometry and stellar luminosity/mass distribution of such streams. This will not only put our Milky Way and M31 in context but, for the first time, also provide an extensive statistical basis for comparison with state-of-the-art, self-consistent cosmological simulations of this phenomenon.
\end{abstract}

\section{Introduction}

Within the hierarchical framework for galaxy formation (e.g., White \& Rees 1978), the stellar bodies of galaxies are expected to form and evolve through dark-matterdriven mass in-fall and successive coalescence of smaller, distinct sub-units that span a wide mass range. Mergers of initially bound sub-halos (which we refer to as satellites; they consist of dark matter, gas, and in most cases stars) are effected by dynamic friction, either through gradual orbital decay or by a single encounter (de-

David Martinez-Delgado, Ramon y Cajal fellow Instituto de Astrofisica de Canarias, e-mail: ddelgado@iac.es 
pending on the initial orbit), its eccentricity and the satellite-to-main-galaxy mass ratio. It is likely that a satellite becomes disrupted by the tidal forces of the larger companion before its orbit spirals all the way to the center. If such a tidal disruption is complete, and no bound satellite is left, dynamical friction ceases to act. If the disruption is only partial at this epoch, the surviving satellite fragment displays extensive tidal tails, leading and trailing its current position in the galactic halo.

While in $\Lambda$-Cold Dark Matter $(\Lambda \mathrm{CDM})$ the interaction rate is expected to drop to the present-day epoch, such disruption of satellites should still occur around normal spiral galaxies. The fossil records of these merger events may be detected nowadays in the form of distinct coherent stellar structures in the outer regions of massive systems. The most spectacular cases of tidal debris are long, dynamically cold stellar streams from a disrupted dwarf satellite, which have wrapped around the host galaxy's disk and roughly trace the orbit of the progenitor satellite. The now wellstudied Sagittarius tidal stream surrounding the Milky Way (Majewski et al. 2003) and the giant stream in Andromeda galaxy (Ibata et al. 2007) are archetypes of these satellite galaxy merger 'fossils' in the Local Group. They provide sound qualitative support for the scenario that tidally disrupted dwarf galaxies are important contributors to stellar halo formation in the Local Group spirals.

State-of-the-art, high-resolution numerical simulations of galaxy formation, built within the $\Lambda$ CMD context (e.g. Moore et al, 1999; Springel et al. 2008), can guide the quest for observational signatures of such star-streams (e.g. Bullock \& Johnston 2005; Johnston et al. 2008). Recent simulations have demonstrated that the characteristics of substructure currently visible in the stellar halos are sensitive to the last (0-8 Gyr ago) merger histories of galaxies, a timescale that corresponds to the last few to tens of percent of mass accretion for a spiral galaxy like the Milky Way. While stellar streams in the Milky Way and Andromeda can be studied in detail, comparison with cosmological models is limited by 'cosmic variance'. However, the current models imply that a survey of 50-100 parent galaxies reaching to a surface brightness of 30 mag arcsec ${ }^{-2}$ would reveal many tens of tidal features, perhaps nearly one detectable stream per galaxy (Johnston et al. 2008). However, a specific comparison of these simulations with observations is missing because no suitable data sets exist. Such a comparison, which could quantify the present subhalo merger rate, is not only important as a test of $\Lambda \mathrm{CDM}$ models, but also as a more direct probe of how resilient disks are to minor mergers.

\section{Stellar tidal streams in external galaxies}

Galactic archeology by looking at tidal remnants is a relatively new field of research that so far has been primarily focused on the Local Group spiral galaxies. The first known tidal stream surrounding the Milky Way (the Sagittarius tidal stream) was discovered less than one decade ago (Mateo et al. 1998; Martínez-Delgado et al. 2001). In recent years, studies have focused on the formation and evolution of our Galaxy have been revolutionized by the first generation of wide-field, digital imag- 

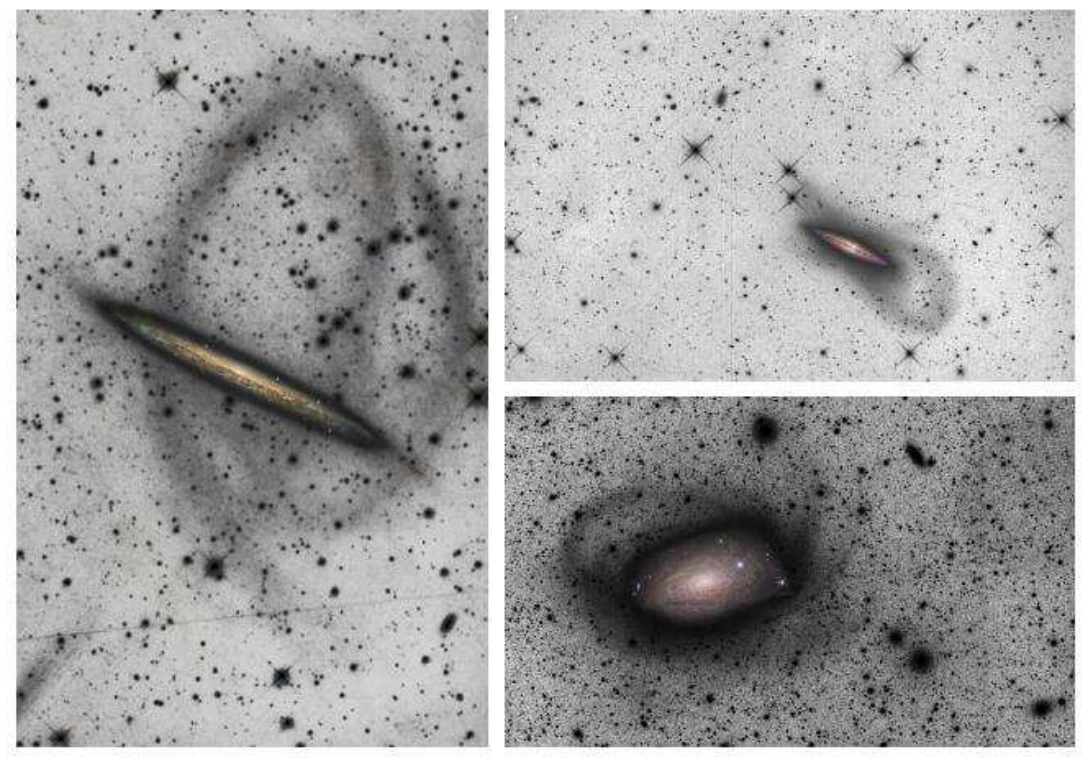

Fig. 1 (left) Deep image of the stellar tidal stream around NGC 5907 obtained with the 0.5 -meter Black Bird Observatory (BBO) telescope (Martinez-Delgado et al 2008a). A N-body model of this structure is shown in Fig. 2; (right, top) A low-galactic latitude stellar tidal stream of NGC 4013, discovered by our team from deep images taken with the BBO telescope; (right, bottom) Deep images taking with a FSQ-106ED telescope of only $10 \mathrm{~cm}$ aperture allowed the discovery of a giant tidal stream in the halo of the spiral galaxy Messier 63 (Martinez-Delgado et al. 2009, in preparation). A colour inset of the disk of each galaxy has been inserted with reference purpose.

ing surveys. The resulting extensive photometric databases have provided, for the first time, spectacular panoramic views of Milky Way tidal streams ( e.g. the Field of Streams: Belokurov et al. 2006) and have revealed the existence of large stellar sub-structures in the halo (Newberg et al. 2002; Juric et al 2008), which have been interpreted as observational evidence of our home Galaxy's hierarchical formation. The discovery of the Monoceros tidal stream (Yanny et al 2003) and the possible Canis Major dwarf galaxy (Martin et al. 2004; Martínez-Delgado et al. 2005), located close to the Galactic plane, indicates that minor mergers might play a relevant role in the formation of the outer regions of spiral disks (Peñarrubia et al. 2006). A multitude of tidal streams, arcs, shells and other irregular structures that are possibly related to ancient merger events can be seen in deep panoramic views of the Andromeda halo (Ibata et al. 2007). These pictures show in detail the level of stellar sub-structure that might be present in the halos of nearby external spiral galaxies. 
Our current understanding of the Local Group spirals has provided a couple of firsthand examples of individual minor mergers and their link to the current state of massive galaxy building (Johnston et al. 2008). The search for analogues to these galactic fossils beyond the Local Group is required not only to see whether the Milky Way and Andromeda galaxies are 'typical' with regard to substructure formation, but to estimate the fractional contribution of accreted mass and the mass spectrum of accreted bodies in the life of these massive systems, an issue that remains unresolved.

Unfortunately, over the past decade only a few cases of confirmed stellar tidal streams have been detected in spiral galaxies outside the Local Group (e.g. Pohlen et al. 2004 and references therein). The first cases of extragalactic tidal streams were reported a decade ago by Malin \& Hardlin (1997). Using special contrast enhancement techniques on deep photographic plates, they were able to highlight two possible tidal streams surrounding the galaxies M83 and M104. Then, deep CCD images of the nearby, edge-on galaxy NGC 5907 by Shang et al. (1998), revealed an elliptically-shaped loop in the halo of this galaxy. This was the most compelling example of a non-Local-Group tidal stream up to now. More recently, very deep images (Martinez-Delgado et al. 2008a,b) have clearly revealed large scale, complex structures of arcing loops in the halos of several nearby galaxies (see Fig. 1). These detailed observations provide an elegant example of how a single, current epoch, low-mass satellite accretion can produce a very complex, rosette-like structure of debris dispersed in the halo of its host galaxy.

\section{A pilot survey of stellar tidal stream in nearby galaxies (2006-2008)}

We have initiated a pilot survey of stellar tidal streams in a select number of nearby, edge-on spiral systems using modest (0.1-0.5-meter), robotic telescopes operating under very dark skies. The main results of this first observational effort are given below.

\subsection{The tidal stream of NGC 5907}

In summer 2006, we re-observed the tidal stream of NGC5907 as a commissioning target to demonstrate the sensitivity of our small aperture telescope for detecting extragalactic tidal streams. Our deep observations showed for first time an interwoven, rosette-like structure of debris dispersed in the halo of this spiral galaxy (Fig.1; Martinez-Delgado et al. 2008a). Its presence provides confirmation that these tidal remnants can survive several gigayears, as predicted by $\mathrm{N}$-body simulations of tidally disrupted stellar systems around the Milky Way (e.g., Law et al 2005; Peñarrubia et al. 2005). 


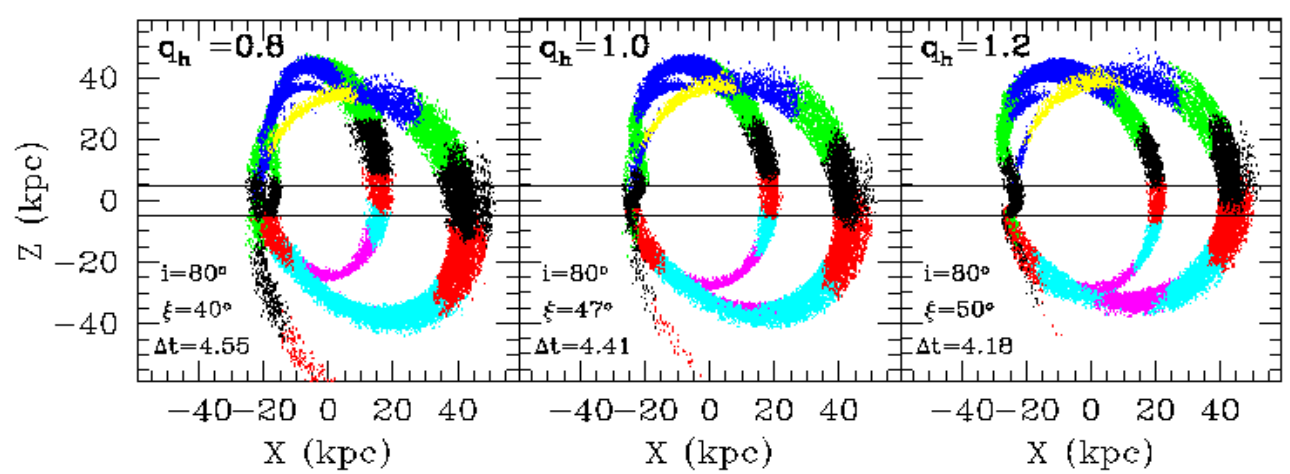

Fig. 2 N-body model of the stellar stream detected in NGC 5907. The satellite is realized as a King model with an initial mass, King core and tidal radii of $M=2 \times 10^{8} M_{\odot}, r_{c}=0.39 \mathrm{kpc}$ and $r_{t}=2.7 \mathrm{kpc}$, respectively (see Martínez-Delgado et al. 2008a for details). For this particular model the orbital period is $T_{r}=0.9 \mathrm{Gyr}$. Each panels adopt a different halo minor-to-major axis-ratio $\left(q_{h}\right)$. Different colors denote different ranges of projected radial velocities, namely yellow (-210,$150) \mathrm{kms} / \mathrm{s}$; blue $(-150,-90) \mathrm{km} / \mathrm{s}$; green $(-90,-30) \mathrm{km} / \mathrm{s}$; black $(-30,+30) \mathrm{km} / \mathrm{s}$; red $(+30,+90) \mathrm{kms}$; cyan $(+90,+150) \mathrm{km} / \mathrm{s}$ and magenta $(+150,+210) \mathrm{km} / \mathrm{s}$. Note that the three models show similar variations of the projected radial velocity along the stream. This result suggests that additional kinematical data may provide insufficient information to break the model degeneracies discussed in Martinez-Delgado et al. (2008a).

Our N-body simulations (Fig.2) of the tidal disruption of a dwarf satellite by a disk galaxy and its dark halo potential suggest that most of the tidal features observed in NGC 5907 can be explained by a single accretion event. Interestingly, this model finds that the stellar stream may be relatively old with the fainter, outer loop material becoming unbound at least 3.6 Gyrs ago. The stellar stream around NGC 5907 may therefore represent one of the most ancient tidal debris ever reported in the halo of a spiral galaxy. It also confirms that spiral galaxy halos in the Local Universe still contain a significant number of galactic fossils from their hierarchical formation and that they can be detected with modest instruments.

\subsection{Discovery of stellar tidal streams in warped disk galaxies}

Promising galaxies in the hunt for extragalactic tidal streams are those that display outstanding asymmetries in optical or HI images. It has been long suggested that these perturbations are a result of gravitational interaction with nearby companions. The most striking case is NGC 4013, an isolated spiral galaxy famous for having one of the most prominent HI warps detected so far (Bottema et al. 1987). Our deep images of this galaxy (Martínez-Delgado et al. 2008b) revealed a faint loop-like structure that appears to be part of a gigantic, low-inclination stellar tidal stream 

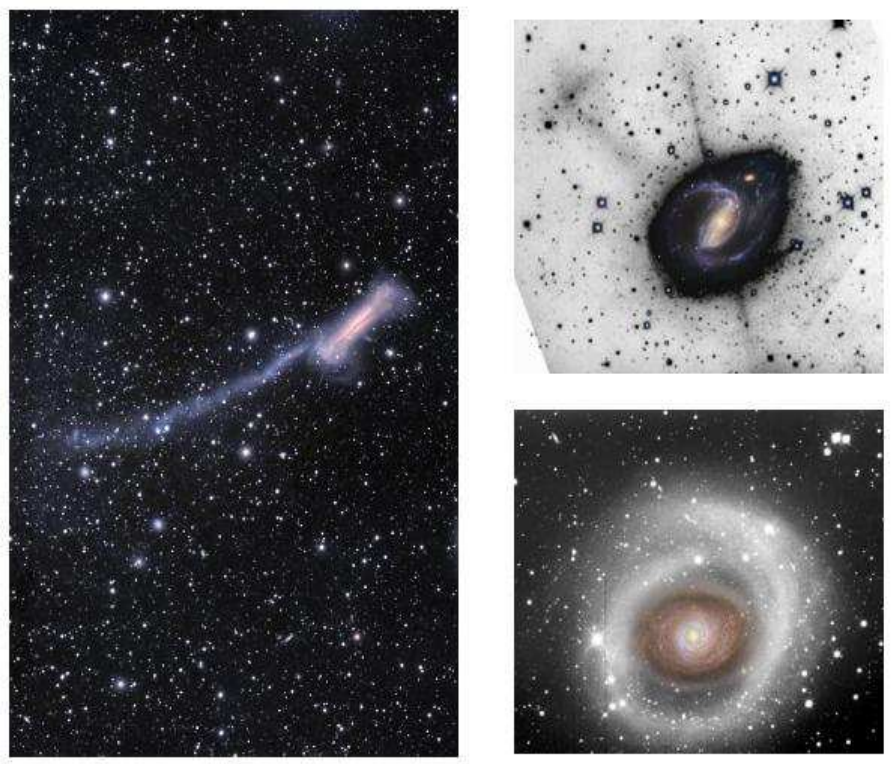

Fig. 3 Diffuse light, giant structures detected in the outskirts of several nearby galaxies in our pilot survey: (left) Panoramic view of the halo of NGC 3628 obtained with a 10-cm telescope. In addition to the known giant filament (Chromey et al. 1998), our deep images reveal for first time new arc-like features in the edge of its perturbed disk, which suggest that this galaxy could be suffering a tidal encounter with a dwarf satellite; (bottom, right) A striking, very faint ring structure in M94 detected with the BBO telescope (Trujillo et al. 2009, in prep.), consistent with being the optical counterpart of the ultraviolet extended disk discovered from GALEX observations (Gil de Paz et al. 2007); (top, right) First wide-field CCD panoramic image of the mysterious jet-like features in the halo of the active galaxy NGC 1097, previous reported in photographic plates (Arp 1976). The tidal origin of these features is still controversial (Higdon \& Wallin 2003).

(Fig. 1). Although its true three dimensional geometry is unknown, the sky- projected morphology of this structure displays a remarkable resemblance to the theoretical predictions for an edge-on view of the Milky Way's Monoceros tidal stream (Peñarrubia et al. 2005). This suggests that the progenitor system (whose current position and final fate remains unknown) may have been a galaxy with an initial mass $\sim 10^{8} M_{\odot}$ moving on a low-inclination $\left(\simeq 25^{\circ}\right)$, nearly-circular orbit. Using this model as a template, the tidal stream may be approximately $\sim 3$ Gyr of age.

We have also discovered a stellar tidal stream in the halo of the nearby spiral Messier 63 (Martinez-Delgado et al. 2009, in preparation). Our data, collected from different telescopes, reveals an enormous, arc-like structure around this galaxy's disk extending $\sim 29 \mathrm{kpc}$ from its center (Fig.1), apparently tilted with respect to its strong gaseous warp. This unassailable indication of a past merger event provides 
an additional example of apparently isolated galaxies with significantly warped gaseous disks that also show evidence indicating the ongoing tidal disruption of a dwarf companion.

These results highlight the fact that disks that appear to be undisturbed in high surface brightness optical images but warped in HI maps can reveal complex signatures of recent accretion events when viewed in deep optical surveys. Additionally, with the growing number of examples of spirals (NGC 5907, NGC 4013, M63) showing a connection between warped disks and evidence of mergers, the origin of galactic warps via interactions with minor mergers is a possibility worth further investigation.

\subsection{Diffuse light structures in nearby spiral galaxies}

Over the last two years, we have also obtained wide field, follow-up images of a dozen familiar, nearby spiral galaxies that are widely known to have diffuse light features in their outer regions. Although our current data for them is not yet deep enough yet (with an estimated surface brightness limit of $\sim 28 \mathrm{mag} / \mathrm{arcsec}^{2}$ ), our panoramic views of their halos have revealed their fossil structures in detail. The most conspicuous cases detected during this observational effort are shown in Fig. 3. In addition, we have also discovered striking stellar tidal streams of different morphological types in several neighboring galaxies (Martinez-Delgado et al. 2009, in preparation). Thus, our deep images are very effective in revealing a plethora of very faint morphological perturbations and dynamical features in the external regions of nearby galactic disks, presumably all signposts of minor gravitational interactions.

\section{Future work}

The promising results of our foray into a more systematic look for tidal streams in the nearby universe encourage a more aggressive attention to this new way of understanding galaxy formation. The overall goal of this project is to conduct the first systematic survey of stellar tidal streams for $\sim 50$ nearby spiral galaxies $(D<15$ $\mathrm{Mpc}$ ) to a (large area) surface brightness sensitivity of $\sim 30 \mathrm{mag} / \mathrm{sqasec}$, in order to obtain the first comprehensive census of such structures in the Local Volume. Based on theoretical predictions on stream counts (one stream expected per galaxy; see Sec.1), we expect this observational effort to yield a tidal stream sample with sufficient statistical significance to undertake a direct comparison with high resolution cosmological simulations of hierarchical galaxy formation and satellite dark matter halo dynamics.

A number of astrophysical problems can be tackled with the data produced from this survey and covering a large variety of classical topics (stellar populations, for- 
mation of galactic disk, galactic dynamics, globular cluster formation, dark matter halo flattening; cosmology; see a discussion in Martínez-Delgado et al. 2008a). The survey will result in estimates of the incidence, size/geometry and stellar luminosity/mass distribution of such streams. The results of the project will provide a direct and stringent test of hierarchical structure formation on this scale, will constrain the present-epoch (minor) interaction rate and probe the minor-merger resilience of stellar disks.

Acknowledgements The authors gratefully thank the following astrophotographers whose collaboration helped make our initial survey possible: Ray Gralak (Fig. 2, M63), Steve Mandel (Fig. 3, NGC 3628), Kenneth Crawford and Mischa Schirmer.

\section{References}

1. Arp, A. 1976, ApJ, 207, 147

2. Belokurov, V., et al. 2006, ApJ, 642, L137

3. Bottema, R., Shostak, G. S., \& van der Kruit, P. C. 1987, Nature, 328, 401

4. Bullock, J. S. and Johnston, K. V.., 2005, ApJ, 635, 931

5. Chromey, F. R., Elmegreen, D. M., Mandell, A., McDermott, J. 1998, AJ, 115, 2331

6. Gil de Paz, A. et al. 2007, ApJS, 173, 185

7. Higdon, J. L. \& Wallim, J. F. 2003, ApJ, 585, 281

8. Ibata, R., Martin, N. F., Irwin, M., Chapman, S., Ferguson, A. M. N., Lewis, G. F., \& McConnachie, A. W. 2007, ApJ, 671, 1591

9. Johnston, K. V., Bullock, J. S., Sharma, S., Font, A., Robertson, B. E., Leitner, S. N. 2008, ApJ, in press (arXiv:0807.3911)

10. Juric, M., et al. 2008, ApJ, 673, 864

11. Law, D. R., Johnston, K. V., \& Majewski, S. R. 2005, ApJ, 619, 807

12. Majewski, S. R., Skrutskie, M. F., Weinberg, M. D., \& Ostheimer, J. C. 2003, ApJ, 599, 1082

13. Malin, D. \& Hadley, B. 1997, PASA, 14,52

14. Martin, N. F., Ibata, R. A., Bellazzini, M., Irwin, M. J., Lewis, G. F., \& Dehnen, W. 2004, MNRAS, 348, 12

15. Martínez-Delgado, D., Aparicio, A., Gómez-Flechoso, M. Á., \& Carrera, R. 2001, ApJ, 549, L199

16. Martínez-Delgado, D., Butler, D., Rix, H. W., Franco, I. V., Peñarrubia, J., Alfaro, E. J., Dinescu, D. I. 2005, ApJ, 633, 205

17. Martínez-Delgado, Peñarrubia, J., Gabany, R. J., Trujillo, I.,Majewski, S. R., Pohlen, M. 2008a, ApJ, 689, 184

18. Martínez-Delgado, Pohlen, M., Gabany, R. J., Majewski, S. R., Palma, C. 2008b, ApJ, in press

19. Mateo, M., Olszewski, E. W., Morrison, H. L. 1998, ApJ, 508, 55

20. Moore, B., Ghigna, S., Governato, F., Lake, G., Quinn, T., Stadel, J., \& Tozzi, P. 1999, MNRAS, 304, 465

21. Newberg, H. J., et al. 2002, ApJ, 569, 245

22. Peñarrubia, J., et al. 2005, ApJ, 626, 128

23. Peñarrubia, J., McConnachie, A., Babul, A. 2006, ApJ,650, L33

24. Pohlen, M., Martínez-Delgado, D., Majewski, S., Palma, C., Prada, F., \& Balcells, M. 2004, in Satellites and Tidal Streams, PASP, 327, 288

25. Shang, Z., et al. 1998, ApJ, 504, L23

26. Springel et al.,2008, MNRAS, in press (arXiv0809:0898)

27. White, S. D. M. \& Rees, M. J. 1978, MNRAS, 183, 341

28. Yanny, B., et al. 2000, ApJ, 540, 825 